\title{
Instrumenten-Tracking im OP-Saal
}

\section{Rudolf Stumberger}

PD Dr. phil., Soziologe und Publizist, Münchner Pressebüro

Die Erfindermesse in Nürnberg zeigt jährlich einen bunten Reigen an neuen Ideen. Die «iENA 2015», die internationalen Fachmesse «Ideen - Erfindungen - Neuheiten» fand Ende Oktober 2015 zum 67. Mal statt. Gezeigt wurden rund 700 Erfindungen, teilgenommen hatten Personen, Verbände und Organisatoren aus 36 Ländern. Es gab Kurioses zu sehen wie ausziehbare Kinderschuhe aus Taiwan. Oder Praktisches wie ein Gerät zum Öffnen der Kokosnuss. Und eine Gruppe von Schülern beschäftigte sich gar mit Medizintechnik im Operationssaal.

Die metallenen Dinge, die unter dem Neonlicht auf dem Tisch liegen, haben Namen wie Organfasszange, Wundspreizer, Bauchdeckenhalter oder Ligaturklemme. Sie werden von den Chirurgen im Operationssaal benutzt - und manchmal im Körper des Patienten vergessen. Das soll in Deutschland immerhin dreitausendmal pro Jahr geschehen, heisst es beim deutschen gemeinnützigen «Aktionsbündnis Patientensicherheit». Damit das in Zukunft nicht mehr passiert, haben sich drei Schüler des Gymnasiums in der baden-württembergischen Kleinstadt Spaichingen hingesetzt und getüftelt. Das Ergebnis ihres Forschergeistes präsentierten sie nun auf der Erfindermesse in Nürnberg.

$\mathrm{Zu}$ besichtigen waren die Messe-Neuheiten in der Halle 12, dort hatten auch Fabian Glaser (17) Christoph Moser (18) und Lukas Ruf (16) ihre Erfindung in Sachen Operationsbesteck auf dem Messestand aufgebaut. Und wie soll verhindert werden, dass Skalpell und Zange statt

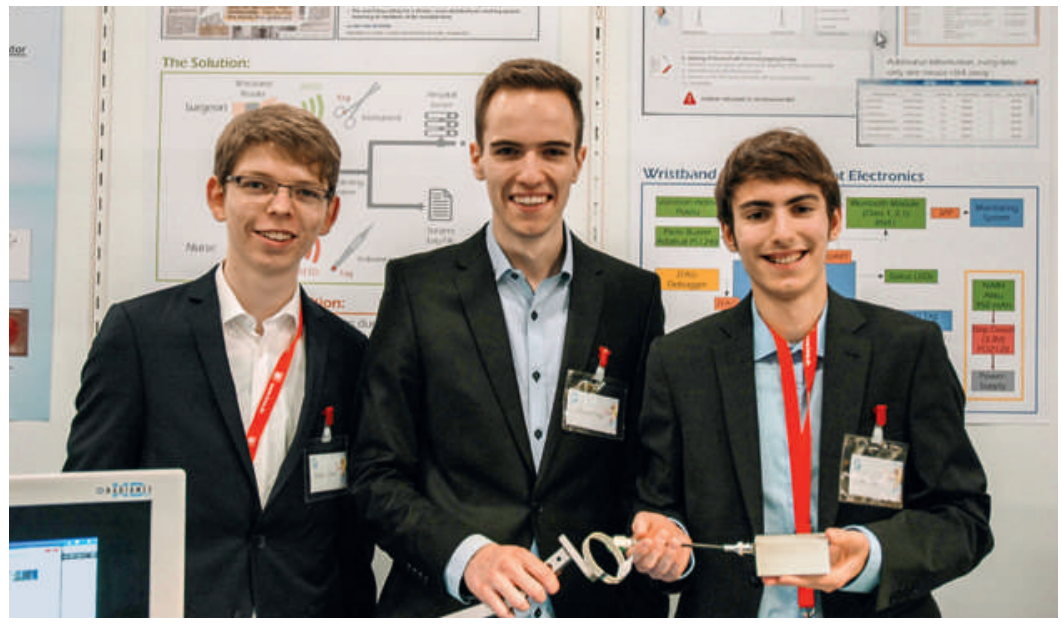

Diese jungen Erfinder haben für ihr Operationsbesteck die Goldmedaille der internationalen Jury der iENA erhalten. in der Desinfektion im zugenähten Bauch des Patienten landen? Der Clou besteht - wie inzwischen in vielen Bereichen - in der Digitalisierung der Instrumente. Das heisst, Organfasszange und Wundspreizer werden mit kleinen sogenannten RFID-Tags versehen. RFID steht für radio-frequency identification, übersetzt etwa «Identifizierung mit Hilfe elektromagnetischer Wellen». Dabei geht es um eine Technologie für Sender-Empfänger-Systeme zum automatischen und berührungslosen Identifizieren und Lokalisieren von Objekten und auch Lebewesen mit Radiowellen. Ein RFID-System besteht aus einem Transponder, der kleiner als ein Reiskorn ist, in die Operationsinstrumente integriert wird

\section{Mit der digitalen Kenntlichmachung}

der Instrumente öffnet sich ein grosses Feld von Anwendungen.

und einen kennzeichnenden Code enthält. Dazu kommt ein Lesegerät zum Auslesen dieser Kennung. Mit dieser digitalen Kenntlichmachung der Instrumente öffnet sich ein grosses Feld von Anwendungen. Mit dem Lesegerät können Chirurgen und Operationsschwestern genau erfassen, wo sich welches Instrument befindet. Die vergessene Schere im Bauchraum gehört damit der Vergangenheit an. Aber mit dem Instrumenten-Tracking lassen sich auch andere Aufgaben lösen. So ist nicht nur eine digitale Dokumentation der Operation mit der Reihenfolge der eingesetzten Instrumente möglich, sondern die Klinik verfügt so auch über eine Übersicht, wie oft die einzelnen Instrumente benutzt werden. Und wie kommen Schüler auf die Idee, sich mit diesem Thema auseinanderzusetzen? "Bei uns in der Region befassen sich viele Firmen mit Medizintechnik», sagt Tüftler Christoph Moser. Bei Führungen durch die Betriebe seien sie auf diese Idee gekommen. Selbst entwickelt haben die drei Gymnasiasten dabei die Platinen des Lesegerätes, der Entwicklungsprozess hat ein Jahr gedauert. Angesiedelt war ihr Projekt beim Schulforschungszentrum in Tuttlingen, dort können Schüler aller Schularten ausserhalb des Schulalltages ihre Ideen verfolgen. Für die drei Medizin-Tüftler vom Gymnasium in Spaichingen steht der nächste Schritt jedenfalls schon fest: Sie wollen bei einer Operation mal live dabei sein, um die Anwendung der Instrumente näher kennenzulernen. Über mangelnde Anerkennung können 


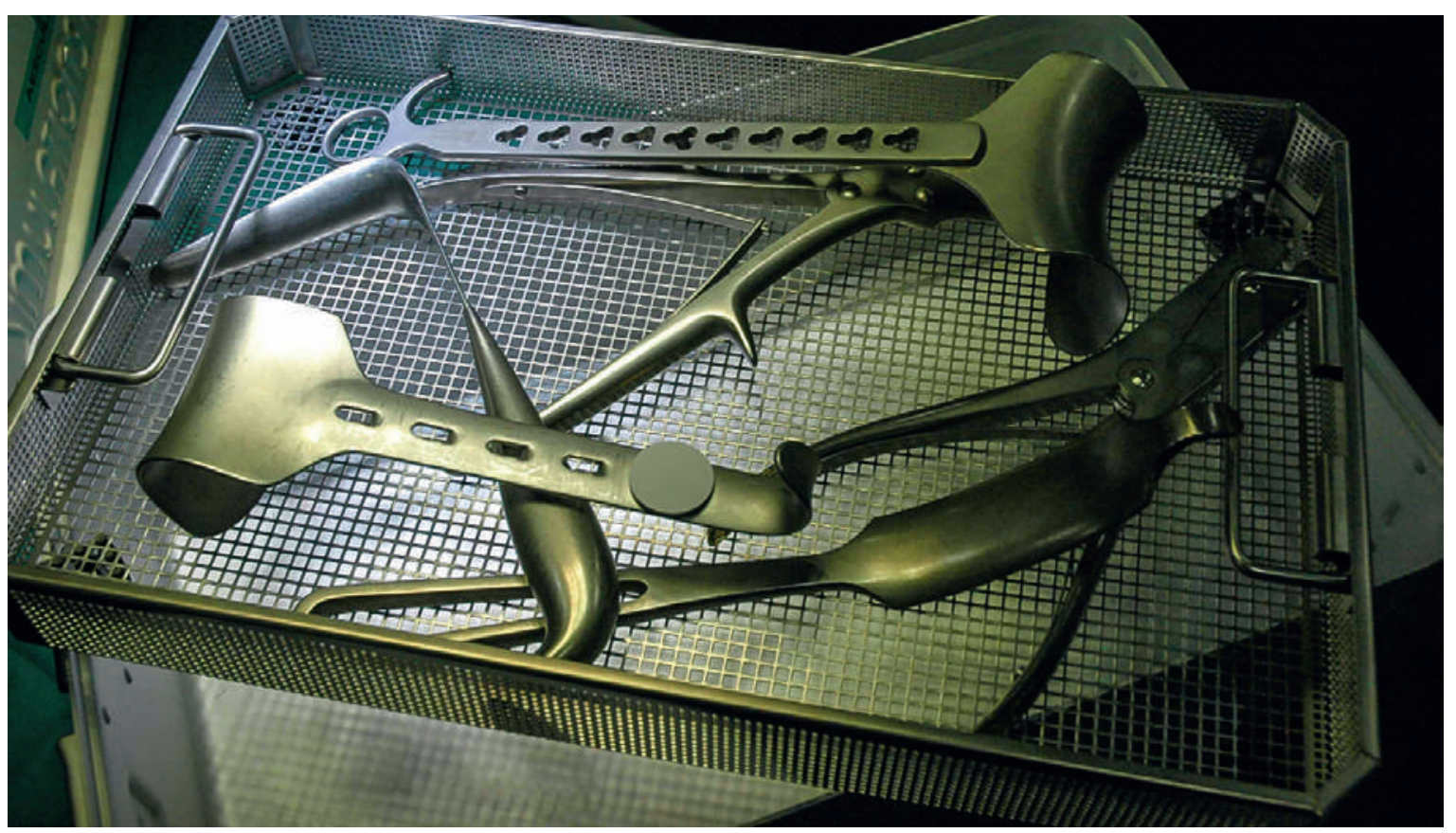

In diesem Operations-Besteck befindet sich ein Lesegerät, das mit elektromagnetischen Wellen erfasst, wo sich welches Instrument befindet.

sich die Schüler jedenfalls nicht beschweren: Immerhin wurde ihnen die Goldmedaille durch die internationale Jury der iENA und der grosse Preis des deutschen Erfinderverbandes verliehen. Das Team will jetzt am Wettbewerb "Jugend forscht» 2016 teilnehmen.

Und haben sich bereits Hersteller oder Kliniken bei den drei Erfindern gemeldet? Konkret gebe es zurzeit einen Optionsvertrag mit der Aesculap AG Tuttlingen für eine Erfindungsübertragung, so die Antwort. Ob Aesculap diese Option ziehe, sei aber unklar. Falls nicht, will das Tüftler-Team 2016 nach weiteren Interessenten suchen. Allerdings testen auch einige Firmen bereits seit einiger Zeit in Kooperation mit grossen Kliniken die Verwendung von RFID-Chips in OP-Instrumenten, Tupfern und Tüchern. Zum Beispiel das Fraunhofer-Institut für Fertigungstechnik und Angewandte Materialforschung. Das Ziel der Feldstudien ist, während einer Operation grafisch auf einem Monitor eine Kontrolle sichtbar zu machen, wie viele Fremdkörper noch im OP-Gebiet liegen und ob am Ende der OP alles, was an den Tisch gebracht wurde, wieder von ihm ent- fernt ist (und damit nicht im Patienten bleibt). Das ist auch den jungen Forschern bekannt: «Wir haben mitbekommen, dass sich auch andere Unternehmen seit längerem mit dieser Herausforderung beschäftigen, dass es aber unseres Wissens nach noch keine Lösung gibt», so Lukas Ruf. Denn diese Technologie hat auch Nachteile, zum Beispiel die Abhängigkeit von Umgebungsbedingungen. So haben Personen und metallische

Die vergessene Schere im Bauchraum gehört damit der Vergangenheit an.

Objekte einen grossen Einfluss auf Fehldetektionen und ausbleibende Detektionen, dieser Einfluss kann nicht gänzlich verhindert werde. So jedenfalls das Ergebnis einer Doktorarbeit an der Universität Leipzig von 2015 zur «Perspektivenorientierte Erkennung chirurgischer Aktivitäten im Operationssaal».

Bildnachweis

Rudolf Stumberger 